
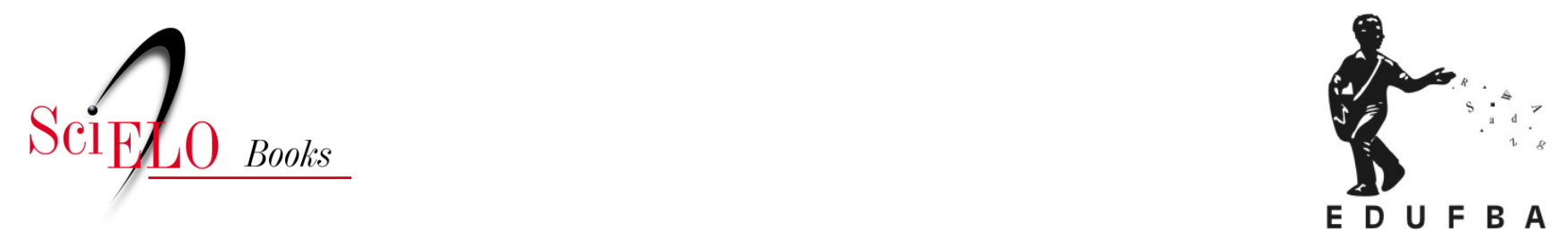

\title{
Nanotecnologia e sua importância no contexto brasileiro
}

\author{
Valdinéia Barreto Ferreira
}

\section{SciELO Books / SciELO Livros / SciELO Libros}

FERREIRA, V.B. Nanotecnologia e sua importância no contexto brasileiro. In: E-science e políticas públicas para ciência, tecnologia e inovação no Brasil [online]. Salvador: EDUFBA, 2018, pp. 97106. ISBN: 978-85-232-1865-2. https://doi.org/10.7476/9788523218652.0007.

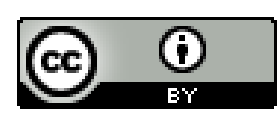

All the contents of this work, except where otherwise noted, is licensed under a Creative Commons Attribution $\underline{4.0 \text { International license. }}$

Todo o conteúdo deste trabalho, exceto quando houver ressalva, é publicado sob a licença $\underline{\text { Creative Commons }}$ Atribição 4.0. 


\section{NANOTECNOLOGIA E SUA IMPORTÂNCIA NO CONTEXTO BRASILEIRO}

Demoramos muito tempo para aprender que tudo na natureza é feito de átomos, agora estamos aprendendo a fazer tudo a partir dos átomos.

$\left(\right.$ Cylon Silva) ${ }^{1}$

A Ciência e a Tecnologia desempenham um papel transformador na sociedade. A Nanociência e a Nanotecnologia $(\mathrm{N} \& \mathrm{~N})$ possuem papéis semelhantes e não menos transformadores. A sugestão de que estes termos sejam fundidos e passem a ser denominados de nanotecnociência é uma prerrogativa que Schulz (2008) apresenta. São campos emergentes da atividade científica e atuam com a manipulação de estruturas em esferas nanométricas ${ }^{2}$ como as moléculas e os átomos. Entretanto, têm causado na sociedade contemporânea imensa repercussão, por representarem respectivamente o estudo e a aplicação tecnológica realizadas em escala nanométrica.

A Nanociência e a Nanotecnologia são associadas a termos como revolução invisível, miniaturização de estruturas, revolução industrial, admirável mundo novo, ficção científica, ciência e tecnologia dos materiais, entre ou-

1 A autoria desta frase é atribuída a Cylon Gonçalves da Silva conforme Shulz (2008, p. 2).

2 A escala nanométrica refere-se à medida que tem por base o nanômetro $(\mathrm{nm})$ que equivale à bilionésima parte de um metro. 
tros. Dos materiais produzidos com a aplicação da nanotecnologia derivam estruturas como: cristais sintéticos diversos, desde diamantes até os cristais de silício com os quais se fabricam os chips dos computadores; materiais magnéticos para gravação e armazenamento de dados; ligas diversas para a indústria mecânica; materiais plásticos e vítreos; e ainda grande variedade de materiais com propriedades específicas e otimizadas para aplicações industriais diversas (CHAVES, 2002).

A meta de dominar parte, pequena que seja, do virtuosismo da natureza na organização da matéria átomo por átomo, molécula por molécula é atribuído à $\mathrm{N} \& \mathrm{~N}$, como destaca Chaves (2002). Apesar do caráter de atualidade que encobre os estudos na área, desde a antiguidade existem indícios da suspeita da existência de estruturas bem pequenas, as nanoestruturas. Essa é uma observação que Santos Junior (2011, p. 23, grifo do autor) resgata ao se referir a N\&N e destacar sua importância no campo científico.

Estamos falando como já apontado [...] da nanociência e da nanotecnologia, que aparecem como o mais amplo salto em direção ao controle e à manipulação de átomos, antigo sonho de muitos cientistas e que já foi capítulo de alguns 'contos de fadas científicos'.

Vale lembrar que desde os tempos de Leucipo de Mileto e Demócrito de Abdera (séc V a.C.) já havia uma suspeita de que a matéria era composta de pequenas estruturas que seriam a sua parte indivisível. Ao contrastar a aparência de um tronco de árvore com as cinzas (produto de uma mesma unidade - a árvore), os atomistas gregos não encontraram nada em comum. Intuíram, então, a existência de uma estrutura capaz de explicar a relação entre os dois elementos. Daí provém o nome átomo, ou melhor, á-tomo, ou seja, aquilo que não pode mais ser quebrado ou dividido em partes.

Resgates outros sobre a utilização de nanopartículas em lugares e contexto histórico passado são pontuados também por Ferreira e Rengel (2009, p. 1861), conforme descreve-se a seguir: 
Os chineses, embora sem ter consciência disso, já aplicavam a nanotecnologia, ao empregarem nanopartículas de carvão em solução aquosa para produzir a tinta nanquim. $\mathrm{Na}$ Europa, o colorido dos vitrais das igrejas medievais, tão ricamente trabalhados pelos artesões, era o resultado da formulação do vidro com nanopartículas de ouro. A famosa Taça de Licurgus, do século IV d.C, que exibe uma cor verde quando a luz é refletida, mas é vermelha sob luz transmitida, é na realidade constituída por nanopartículas de ouro e prata.

A invenção do microscópio de varredura por tunelamento, Scanning Tunneling Microscope (STM), em 1981, por Gerd Binning e Heinrich Roher, do laboratório da IBM em Zurique é considerado como um dos feitos mais importantes para o desenvolvimento da N\&N (CHAVES, 2002). Esse equipamento permite a visão e manipulação das nanoestruturas, um grande passo que impulsionou o deslanchar de uma área emergente, com inserção em vários segmentos da sociedade, um impacto científico e econômico crescente para a população mundial.

Algumas áreas de inserção da Nanotecnologia são pontuadas de modo resumido na Figura 6, e permite vislumbrar a dimensão das aplicações e frentes de atuação que a área congrega. Fica evidente a interdisciplinaridade presente e a contribuição que essa diversidade representa em termos de benefícios para a sociedade. Medicina, Química, Física, Engenharia, Biologia e Informática são apenas alguns exemplos de seu escopo de abrangência.

A prática científica realizada por campos do conhecimento tão distintos passa a fundir-se e derivar produtos que vêm transformando o dia a dia da sociedade contemporânea. Representa implicações que interferem na vida cotidiana, e estão cada vez mais próximas e a infiltrar-se de modo quase invisível nas vidas humanas. Os resultados das aplicações de Nanotecnologia, benéficos ou não, são questões nas agendas de discussões mundiais e o Brasil não pode furtar-se de inserir-se nelas. 
Figura 6 - Áreas de inserção da Nanotecnologia e aplicações

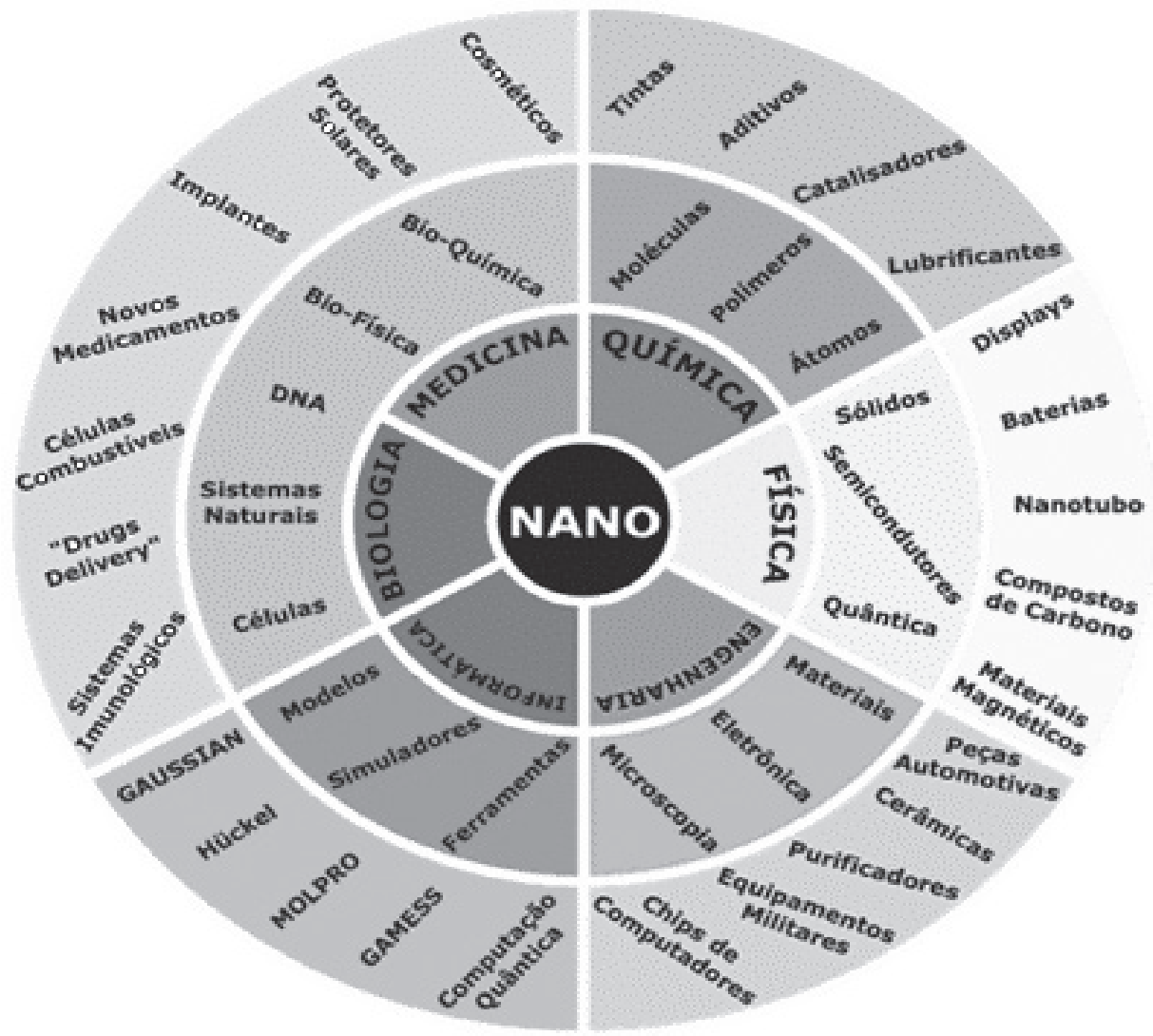

Fonte: Adaptado de Nanotecnologia (2009).

A literatura que cobre a temática está repleta de indicações e detalhes que permitem ampla visibilidade do potencial robusto e detalhado sobre o assunto. No Quadro 5, são pontuadas mais algumas aplicações desta tecnologia tão presente na rotina da sociedade que ainda nem se apercebeu, como deveria, das implicações que ela vem acarretando para sua vida. Uma inserção quase que imperceptível mas consistente e crescente, em segmentos industriais e de serviços, além da medicina, agricultura, segurança entre outros, marcando a revolução que aplicações dessa natureza estão acarretando na sociedade mundial de modo irreverssível. 
Quadro 5 - Aplicações da Nanotecnologia
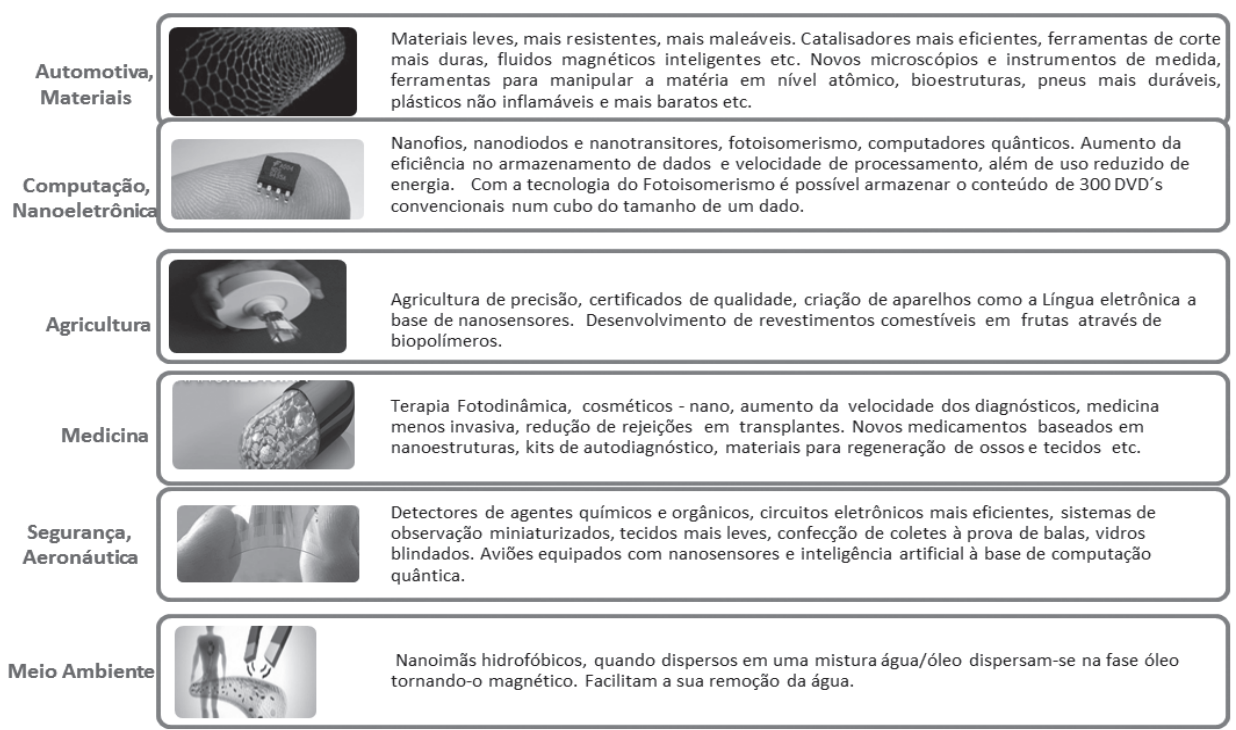

Fonte: Elaboração da autora baseada em Santos Júnior (2011, p. 32).

\section{Mundo das nanoestruturas: conceito, origem e fundamentos da Nanotecnologia}

Nanotecnologia é uma área do conhecimento que causa muita curiosidade e expectativa, especialmente para a parcela da sociedade na qual o tema é pouco difundido. Um mundo desconhecido e cheio de mistérios que pouco a pouco vem sendo desmistificado. É evidente a preocupação em levar para a população uma melhor compreensão.

A etimologia do termo nano, prefixo da palavra nanotecnologia, é atribuída ao grego nanno-, de nános ‘anão', e tem sua origem documentada em vocábulos eruditos, a partir do século XIX (CUNHA, 2011). A este prefixo é creditada a intenção dos cientistas de representarem partículas de magnitude demasiadamente pequenas, os nanômetros (nm), conforme destaca Santos Júnior (2011). Esse autor ainda reitera que o termo "nano também serve como parâmetro de medida, um nanômetro (nm) equivalendo à bilionésima parte de um metro.”

A demonstração da escala nanométrica é apresentada na Figura 7 e ilustra como as nanoestruturas podem ser representadas em dimensões dezenas de 
mil vezes menor do que a do diâmetro de um fio de cabelo, como no exemplo apresentado. Santos Junior (2011, p. 29) acrescenta ainda que "para termos uma noção mais clara, um átomo mede cerca de dois décimos de um nanômetro $(0,2$ nm) e um vírus cerca de 100 nanômetros (100nm)."

Figura 7 - Representação de nanoestruturas em uma escala nanométrica

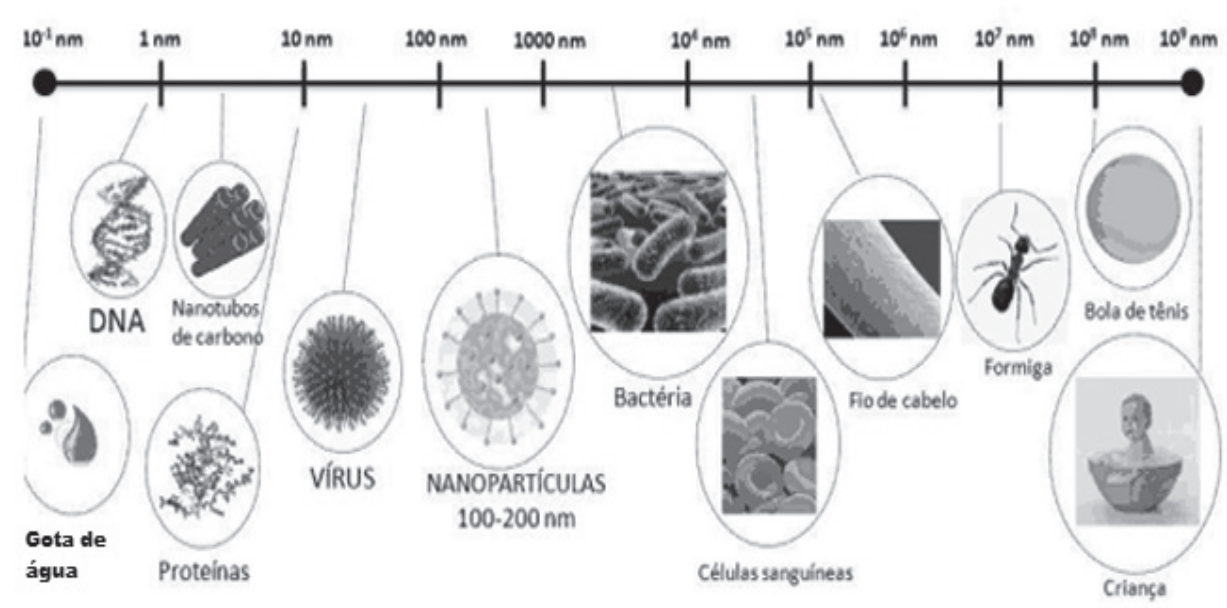

Fonte: Adaptado de TECSAUGEM (2016).

A origem da utilização do termo nanotecnologia possui diversas indicações na literatura científica A palestra proferida por Richard Fernman em 1959, é creditada como marco fundador da área. Ao engenheiro japonês Norio Taniguchi é atribuído à utilização do termo pela primeira vez, em 1974, quando ele o utilizou para designar uma nova tecnologia que ia além do controle de materiais e da engenharia em microescala. Encontam-se também na literatura referências a Eric Drexler como grande disseminador do vocábulo para o grande público, ao atribuir ao termo o significado correspondente à metodologia de processamento que envolve a manipulação átomo a átomo (FERREIRA; RANGEL 2009; SCHULZ, 2013). 
Entretanto, o professor Schultz (2013) conta uma história um pouco diferente $^{3}$ e faz uma incursão a um passado bem mais remoto do que é comumente apresentado nos manuais de introdução à Nanotecnologia, ao referir-se à história da N\&N. Segundo o autor, contar a história da Nanotecnologia sem o devido resgate de suas origens, nada acrescenta a sua compreensão como uma atividade humana de pesquisa e inovação, com importante repercussão social. Contudo, ele ressalva que essa contribuição serve como ponto de partida para a construção de um olhar diferente sobre a temática. A Nanotecnologia é definida conforme Zaneti-Ramos e Creczynski-Pasa (2008, p. 95) como:

[...] a arte de manipular a matéria em nível atômico, construindo moléculas inéditas, com propriedades diferentes. A nanotecnologia encontra aplicações em praticamente todos os setores industriais e de serviços, incluindo as nanopartículas, os revestimentos, catalisadores e nanocomponentes. Muitas destas, já no mercado, revolucionam aplicações de diversos produtos, mudando radicalmente o custo produtivo de muitas indústrias, podendo tornar os produtos não nano alternativas, muitas vezes, não competitivas.

O trabalho realizado de modo incessante por pesquisadores da área contribui de modo satisfatório para atingir resultados que atendem às diversas frentes em que atuam, sejam científicas ou técnicas. No cenário mundial, o despertar para o potencial global da Nanotecnologia criou uma corrida em países, como Estados Unidos e Japão e em conglomerados representados pela União Europeia, em busca do desenvolvimento científico e tecnológico que desse conta da revolução anunciada. Os investimentos em recursos financeiros e humanos para atuar nessa frente de trabalho foram significativos, especialmente da indústria mundial.

São identificadas diversas iniciativas desses países, contudo, os Estados Unidos inicia em 2000, um programa considerado ambicioso pelo montante financeiro empregado. A National Nanotechnology Initiative (NNI) repre-

3 Para maiores detalhes sobre a "história um pouco diferente" consultar o artigo de Schulz (2013). 
senta um programa de pesquisa e desenvolvimento em Nanotecnologia que aumentou as despesas federais em somas altíssimas, um investimento pesado na tecnologia emergente. De modo normativo é assegurada a continuidade do programa pelos anos seguintes de 2005 a 2008 (ZANETI-RAMOS; CRECZYNSKI-PASA, 2008).

O Japão, a China, a Coreia do Sul, Taiwan, Austrália, Canadá, Índia, Malásia, Nova Zelândia, Filipinas, entre outros, são exemplos de países que seguiram os passos dos Estados Unidos e passaram a fazer investimentos governamentais em pesquisas na área de Nanotecnologia. A China também lançou um programa semelhante ao dos Estados Unidos, em 2001, o National Nanotechnology Development Strategy (NNDS), o qual cobria o período de 2001 a 2010. Os estudos desenvolvidos na área realizam-se em um ambiente colaborativo e interdisciplinar, cercado da contribuição global entre países, comunidades profissionais e organizações internacionais (PLENTZ; FAZZIO, 2013; ZANETI-RAMOS; CRECZYNSKI-PASA, 2008).

A movimentação em torno da tecnologia emergente chega ao Brasil. Atualmente, conforme Plentz e Fazzio (2013, p. 23) destacam, "todos os países ditos desenvolvidos e um número grande e crescente de países emergentes e em desenvolvimento têm iniciativas e programas nacionais em $\mathrm{N} \& \mathrm{~N}$, inclusive o Brasil”. As primeiras iniciativas de cunho oficial registradas na área começam no ano de 2000, com reuniões no âmbito do MCT sobre o desenvolvimento futuro da N\&N no país.

Contudo, é importante registrar que em anos anteriores a 2001 havia indícios de pesquisas na área de Nanotecnologia no país, conforme pontua Martins (2007, p. 9) ao afirmar: "sabemos de antemão que já existiam pesquisadores e grupos de pesquisadores trabalhando com nanotecnologia no século passado, bem como diversas teses defendidas sobre o assunto."

A prevalência por formação em redes denota o cunho colaborativo da área e reforça a interdisciplinaridade latente. No Quadro 6, apresenta-se uma breve cronologia dos fatos marcantes para o desenvolvimento da Nanotecnologia no Brasil e destaca-se a ação governamental para o fomento de ações na área. Um desenho dos acontecimentos que evidenciam o crescimento desse domínio do 


\title{
conhecimento no país e o interesse de outros por uma tecnologia considerada estratégica e de vanguarda.
}

\author{
Quadro 6 - Cronologia dos fatos relevantes para a área de Nanotecnologia no \\ Brasil
}

\begin{tabular}{|c|c|}
\hline \multicolumn{2}{|r|}{ FATOS RELEVANTES PARA A ÁREA DE NANOTECNOLOGIA NO BRASIL } \\
\hline 2000 & Reunião seminal do CNPq/MCT sobre o desenvolvimento futuro da N\&N no país. \\
\hline 2001 & $\begin{array}{l}\text { Criadas quatro redes cooperativas de pesquisa na área de Nanotecnologia: Rede de } \\
\text { Nanonotecnologia Molecular e de Interfaces (Renami); Rede de Nanodispositivos, } \\
\text { Semicondutores e Materiais Nanoestruturados (NanoSemiMat); Rede de } \\
\text { Nanobiotecnologia (NanoBioTec); e a Rede de Materiais Nanoestruturados (Nanoest). } \\
\text { Todas essas com fomentos provenientes do CNPq/MCT. Foram também apoiados } \\
\text { quatro Institutos (Virtuais ) do Milênio na área. }\end{array}$ \\
\hline 2002 & $\begin{array}{l}\text { A Capes concede seis bolsas de doutorado para o Laboratório Nacional de Luz } \\
\text { Síncroton (LNLS) em convênio firmado entre a Capes e a Associação Brasileira de Luz } \\
\text { Síncroton (ABTLuS) mantenedora do laboratório. }\end{array}$ \\
\hline 2003 & $\begin{array}{l}\text { Criada a Coordenação-Geral de Políticas e Programas de Nanotecnologia, atualmente } \\
\text { Coordenação de Micro e Nanotecnologias (CGNT). }\end{array}$ \\
\hline 2003 & $\begin{array}{l}\text { Criação do Grupo de Trabalho (GT) pela portaria MCT n² 252, de 16.05.2003, } \\
\text { para elaborar a proposta do Programa de Desenvolvimento da Nanociência e } \\
\text { Nanotecnologia. }\end{array}$ \\
\hline 2004 & $\begin{array}{l}\text { Início do Programa de Desenvolvimento da Nanociência e Nanotecnologia no âmbito } \\
\text { do Plano Plurianual (PPA) } 2004 \text { - } 2007 .\end{array}$ \\
\hline 2004 & $\begin{array}{l}\text { Criação do Grupo de Trabalho para estudo sobre a implantação do Laboratório } \\
\text { Nacional de Micro e Nanotecnologia. }\end{array}$ \\
\hline 2004 & Criada a Ação Transversal de Nanotecnologia nos Fundos Setoriais. \\
\hline 2004 & $\begin{array}{l}\text { Instituída a Rede BrasilNano e seu Comitê Gestor pela portaria n } 614 \text {, de } 1^{\circ} \text { de } \\
\text { dezembro de 2004, composta por dez redes de pesquisa em Nanotecnologia: Rede } \\
\text { de Nanofotônica; Rede Nacional de NanoBiotecnologia e Sistemas Nanoestruturados } \\
\text { (NanoBioEstruturas); Rede de Nanotecnologia Molecular e de Interfaces - Estagio } \\
\text { III; Nanotubos de Carbono: ciência e Aplicações; Nanocosméticos: do Conceito } \\
\text { às Aplicações Tecnológicas; Microscopia de Varredura de Sondas - Software e } \\
\text { Hardware Abertos; Simulação e Modelagem de nanoestruturas; Rede Cooperativa } \\
\text { de Pesquisa em Revestimentos Nanoestruturados; Nanoglicobiotecnologia; Rede de } \\
\text { Nanobiomagnetiso. }\end{array}$ \\
\hline 2005 & Designados os membros do Conselho Diretor da Rede BrasilNano. \\
\hline 2005 & Lançado o Programa Nacional de Nanotecnologia (PNN). \\
\hline 2005 & $\begin{array}{l}\text { Assinado o Protocolo de Intenções entre Brasil e Argentina criando o Centro } \\
\text { Brasileiro-Argentino de Nanotecnologia (CBAN). }\end{array}$ \\
\hline 2005 & $\begin{array}{l}\text { Selecionadas dez Redes Nacionais de Nanotecnologia, com atuação prevista para o } \\
\text { período 2006-2009. }\end{array}$ \\
\hline 2006 & Início de atividades do CBAN. \\
\hline 2007 & $\begin{array}{l}\text { Lançamento do Plano de Ação em C\&T\&I - PACTI, cujas ações são executadas de } \\
\text { forma articulada e coordenada por diversos Ministérios, tendo à frente o MCT. }\end{array}$ \\
\hline
\end{tabular}




\begin{tabular}{|c|c|}
\hline 2008 & $\begin{array}{l}\text { Inauguração do Centro de Nanociência e Nanotecnologia Cesar Lattes, construído no } \\
\text { campus do LNLS, em março de } 2008 \text {. }\end{array}$ \\
\hline 2008 & $\begin{array}{l}\text { Lançamento pelo Governo Federal da Política de Desenvolvimento Produtivo (PDP) } \\
\text { em maio de 2008. Integra a PDP o Programa Mobilizador em Nanotecnologia, cuja } \\
\text { gestão está a cargo do MCT. }\end{array}$ \\
\hline 2008 & $\begin{array}{l}\text { Lançada chamada para o Programa INCTs que contempla as oito áreas estratégicas do } \\
\text { PACTI 2007-2010, dentre elas a de Nanotecnologia. }\end{array}$ \\
\hline $\begin{array}{l}2008 / \\
2009\end{array}$ & $\begin{array}{l}\text { Esforço conjunto entre } \mathrm{MCT}, \mathrm{MDCl} \text { e a Associação Brasileira de Desenvolvimento } \\
\text { Industrial (ABDI) para divulgação da Nanotecnologia no setor privado, ressaltando } \\
\text { sua relevância estratégica como ferramenta para inovação. }\end{array}$ \\
\hline 2009 & $\begin{array}{l}\text { Lançamento do Fórum de Competitividade de Nanotecnologia como ferramenta } \\
\text { estratégica de apoio às iniciativas e programas segundo as dimensões da PDP. } \\
\text { Criação de quatro GTs. }\end{array}$ \\
\hline 2010 & Apoio a 17 novas redes cooperativas através do edital MCT/CNPq n 74/2010. \\
\hline 2011 & $\begin{array}{l}\text { Aberta uma chamada pública MCTI/CNPq n 17/2011. Apoio à criação de } \\
\text { redes cooperativas de pesquisa e desenvolvimento em nanotoxicologia e } \\
\text { nanoinstrumentação. }\end{array}$ \\
\hline 2012 & $\begin{array}{l}\text { Lançamento do Edital MCTI/CNPq n }{ }^{\circ} 16 / 2012 \text { pela CGNT em conjunto com o CNPq, } \\
\text { para apoiar tecnologias inovadoras e/ou aumento da produção, prototipagem de } \\
\text { escala em nanotecnologia e que apoio } 25 \text { projetos com investimento total de pouco } \\
\text { mais de } \mathrm{R} \$ 6 \text { milhões. }\end{array}$ \\
\hline 2013 & $\begin{array}{l}\text { Ação do MEC e da Capes em acordo de cooperação entre Portugal e Espanha na área } \\
\text { de Nanotecnologia }\end{array}$ \\
\hline 2014 & $\begin{array}{l}\text { Lançamento da Chamada INCT-MCTI/CNPq/Capes/FAPs nº } 16 \text { de 2014, em } \\
\text { continuidade ao Programa INCTs. }\end{array}$ \\
\hline 2016 & $\begin{array}{l}\text { Resultado da Chamada INCT-MCTI/CNPq/Capes/FAPs n } 16 \text { de 2014, divulgado no } \\
\text { dia } 11 \text { de maio de } 2016 \text {, com } 230 \text { INCTs pré-aprovados. }\end{array}$ \\
\hline
\end{tabular}

Fonte: Elaboração as autora baseada em dados de pesquisa e informações do CNPq (2016b), Plentz e Fazio (2013).

Após a sinalização de algumas medidas adotadas no âmbito governamental, ficou evidente os esforços despendidos pelo governo em iniciativas que buscavam o desenvolvimento e fortalecimento da Nanotecnologia no país. A pesquisa desenvolvida analisou a $e$-Science e as práticas colaborativas voltadas à inovação e a ela associadas, no âmbito dos INCTs da área de Nanotecnologia. Justificou-se desse modo, a incursão realizada pela pesquisadora, no âmbito das iniciativas para desenvolvimento da $\mathrm{N} \& \mathrm{~N}$, um esforço no sentido de entendimento e apropriação das questões peculiares ao fomento de pesquisas na área. 